\title{
MARCZEWSKI SETS, MEASURE, AND THE BAIRE PROPERTY. II
}

\author{
JOHN T. WALSH
}

(Communicated by R. David Mauldin)

\begin{abstract}
An example is given (in $Z F C$ ) of a hereditarily Marczewski set which does not have the Baire property in the wise sense and is not Lebesgue measurable. It is then shown that Marczewski sets, which do not satisfy the c.c.c., possess a hull property.
\end{abstract}

\section{INTRODUCTION}

This paper is a continuation of the work on Marczewski sets started in [7]. The purpose here is to more clearly establish the relationships between Marczewski sets, sets with the Baire property, and measurable sets. In this paper all spaces considered are uncountable Polish spaces (i.e. complete, separable, and metric) and will usually be referred to as $X$ or $Y$.

In [6] E. Marczewski defined a set $M$ to have property $(s)$ if every perfect set $P$ contains a perfect subset $Q$ such that either $Q \subseteq M$ or $Q \cap M=\varnothing$. Further he defined a set to have property $\left(s^{0}\right)$ if every perfect set $P$ contains a perfect subset $Q$ such that $Q \cap M=\varnothing$. Sets with property ( $s$ ) are commonly referred to as Marczewski sets.

The Baire property in the wide sense will be referred to as property $B_{w}$, and the Baire property in the restricted sense will be referred to as property $B_{r}$. Universally measurable sets will be referred to as sets with property $U$, and universal null sets will be referred to as sets with property $U_{0}$. The classes of sets with property $(s)$, sets with property $\left(s^{0}\right)$, sets with property $B_{w}$, first category sets, sets with property $B_{r}$, always first category sets, universally measurable sets, universal null sets, Lebesgue measurable sets, and Lebesgue measure zero sets will be denoted as $(s),\left(s^{0}\right), B_{w}, F C, B_{r}, A F C, U, U_{0}, \mathscr{L}$, and $\mathscr{L}_{0}$ respectively. More on these classes of sets can be found in [1] and [2]. Also, $C$ will be used to denote the cardinality of the continuum.

Received by the editors October 19, 1988. Presented at the Sixth Annual Auburn Miniconference on Real Analysis, Auburn University, Alabama, April 7, 1989 (Sponsored by NSF).

1980 Mathematics Subject Classification (1985 Revision). Primary 28A05; Secondary 54H05.

Key words and phrases. Marczewski sets, Baire property, measurability, continuum hypothesis, operation $(A)$, c.c.c., hull. 


\section{Main example}

Example 1. There exists (in $Z F C$ ) a set $M \subseteq X \times Y$ with property $\left(s^{0}\right)$ that does not have property $B_{w}$. Furthermore, if $\mu$ and $\nu$ are continuous finite Borel measures on $X$ and $Y$ then $M$ is nonmeasurable with respect to $(\mu \times \nu)$.

Note that if $X \times Y$ is $(0,1) \times(0,1)$ then $M$ would not be Lebesgue measurable in $(0,1) \times(0,1)$. Also note that under the continuum hypothesis such a set is easily constructed by taking the union of a Lusin set and a Sierpinski set. If $D \subseteq X \times Y$ let

$$
U_{X}(D)=\{x \in X \mid(\{x\} \times Y) \cap D \text { is uncountable }\} .
$$

Construction of Example 1. First let $\mathscr{A}=\left\{A \subseteq X \times Y \mid A\right.$ is perfect and $U_{X}(A)$ is uncountable $\}=\left\{A_{\alpha} \mid \alpha<c\right\}$ and note that by [2, p. 496, Theorem 3] $U_{X}\left(A_{\alpha}\right)$ is an uncountable analytic set for each $A_{\alpha} \in \mathscr{A}$. Now for each $\alpha<c$ choose $R c_{\alpha}$ from $U_{X}\left(A_{\alpha}\right)-\left(\bigcup_{\beta<\alpha}\left\{b_{\beta}\right\}\right)$ and let $B_{\alpha}=\left(\left\{b_{\alpha}\right\} \times Y\right) \cap A_{\alpha}$. Note that $\mathscr{B}=\left\{B_{\alpha} \mid \alpha<c\right\}$ is an uncountable collection of disjoint Borel sets, so by [7, Theorem 2.2] there exists a set $M$ with property $\left(s^{0}\right)$ which intersects each member of $\mathscr{B}$ and therefore each member of $\mathscr{A}$. It can be assumed that $M$ intersects each member of $\mathscr{B}$ only once.

The Kuratowski-Ulam theorem [3] implies that all sets in $B_{w}-F C$ contain a member of $\mathscr{A}$ and therefore intersect $M$. So $M$ cannot be first category. Since $M$ is totally imperfect, $M$ cannot be in $B_{w}-F C$ and therefore $M$ cannot have property $B_{w}$. Now suppose $\mu$ and $\nu$ are continuous finite Borel measures on $X$ and $Y$. Fubini's theorem implies that every set of positive measures with respect to $(\mu \times \nu)$ contains a member of $\mathscr{A}$ and therefore intersects $M$. So $M$ cannot have measure zero with respect to $(\mu \times \nu)$. Since $M$ is totally imperfect, $M$ cannot have positive measure with respect to $(\mu \times \nu)$ and therefore cannot be measurable with respect to $(\mu \times \nu)$.

This example entailed the construction, in $X \times Y$, of a collection of disjoint Borel sets, $\mathscr{B}$, such that no first category set and no set of measure zero with respect to $(\mu \times \nu)$ intersects each member of $\mathscr{B}$. In [4] Sierpiński constructed a similar collection of Borel sets in $[0,1]$, such that no Lebesgue measure zero set intersects each member of the collection.

\section{MARCZEWSKi SETS AND Operation $(A)$}

In [5] Marczewski showed the following two theorems involving the closure of classes of sets under operation $(A)$. Here $N(\mathscr{K})$ will be used to denote the sets in the class $\mathscr{K}$ whose subsets are also in $\mathscr{K}$. The first theorem defines a hull condition for classes of sets.

Theorem 1 (Marczewski). If a class of sets $\mathscr{K}$ is a $\sigma$-field and if $E \subseteq X$ there exists a set $K \in \mathscr{K}$ such that $E \subseteq K$ and if $L \in \mathscr{K}$ containing $E$ then $(K-L) \in N(\mathscr{K})$, then $\mathscr{K}$ is closed under operation $(A)$. 
Theorem 2 (Marczewski). If a class of sets $\mathscr{K}$ is a $\sigma$-field and satisfies the c.c.c., then $\mathscr{K}$ is closed under operation $(A)$.

Now in [6] Marczewski was able to use a direct and simple proof to show that sets with property $(s)$ are closed under operation $(A)$, and so he did not need to use his previous theorems. This was pointed out to the author by John C. Morgan, of California State University at Pomona. Here these theorems are used to help show the differences and similarities between some of the classes of sets mentioned earlier.

First it is easy to see that $B_{w}$ and $\mathscr{L}$ satisfy the conditions of both theorems. The set constructed in Example 1 can be used to demonstrate that $B_{r}$ and $U$ do not satisfy the hull condition of Theorem 1 . This follows from the fact that such a set $M$ would have to be in $B_{r}-A F C$ or $U-U_{0}$ and contain a perfect subset not intersecting the set from Example 1. Since any Cantor set can be divided into continuum many disjoint Cantor sets, it is easy to see that $(s)$, $B_{r}$, and $U$ do not satisfy the c.c.c. It is obvious that $(s)$ is a $\sigma$-field, as shown by Marczewski. It will now be shown that $(s)$ satisfies the hull condition of Theorem 1. A set $M$ is Bernstein relative to a perfect set $P$ if both $M \cap P$ and $P-M$ intersect every perfect subset of $P$.

Theorem 3. If $Z \subseteq X$ then there exists a set $S$ containing $Z$ such that $S$ has property (s), and if $P$ is a perfect subset of $X-Z$ then $|P \cap S|<C$.

Proof. Suppose $Z \subseteq X$. Let $\mathscr{P}=\{P \subseteq X \mid P$ is perfect $\}$, let $\mathscr{A}=\{A \in \mathscr{P} \mid Z$ is Bernstein relative to $A$ or $A \subseteq Z\}$, and let $\mathscr{B}=\{B \in \mathscr{P} \mid B \cap Z=\varnothing\}$. Note that if $\mathscr{A}$ is empty, then $Z$ will have property $\left(s^{0}\right)$ and the theorem is trivial. Also note that if $\mathscr{B}$ is empty, the theorem is similarly trivial. Now if both $\mathscr{A}$ and $\mathscr{B}$ are nonempty, then $|\mathscr{A}|=|\mathscr{B}|=C$. Also note that $(\mathscr{A} \cap \mathscr{B})=\varnothing$ and that if $A \in \mathscr{A}$ and $B \in \mathscr{B}$, then $A \cap B$ is at most countable. Further note that if $D \in \mathscr{P}-(\mathscr{A} \cup \mathscr{B})$, then $D$ will contain a perfect subset $D^{\prime} \in(\mathscr{A} \cup \mathscr{B})$. Now let $\mathscr{A}=\left\{A_{\alpha} \mid \alpha<c\right\}$ and $\mathscr{B}=\left\{B_{\alpha} \mid \alpha<c\right\}$. Define $S=Z \cup\left(\bigcup_{\alpha<c}\left(A_{\alpha}-\bigcup_{\beta<\alpha} B_{\beta}\right)\right)=\bigcup_{\alpha<c}\left(\left(A_{\alpha} \cup Z\right)-\bigcup_{\beta<\alpha} B_{\beta}\right)$. Now to show $S$ has property $(s)$ assume $P$ is a perfect set. If $P=A_{\alpha} \in \mathscr{A}$ then $|P-S|=\left|A_{\alpha}-S\right| \leq\left|A_{\alpha} \cap\left(\bigcup_{\beta<\alpha} B_{\beta}\right)\right|<C$, therefore $P$ will contain a perfect subset $Q$ such that $Q \subseteq S$. If $P=B_{\alpha} \in \mathscr{B}$ then $|P \cap S|=\left|B_{\alpha} \cap S\right|=$ $\left|B_{\alpha} \cap\left(\bigcup_{\beta<\alpha} A_{\beta}\right)\right|<C$, so $P$ contains a subset $Q$ such that $Q \cap S=\varnothing$. If $P \in \mathscr{P}-(\mathscr{A} \cup \mathscr{B})$ then $P$ will contain a perfect subset $P^{\prime} \in(\mathscr{A} \cup \mathscr{B})$ and therefore a perfect subset $Q$ such that $Q \subseteq S$ or $Q \cap S=\varnothing$. Therefore $S$ has property $(s)$ and $|S \cap B|<C$ for each $B \in \mathscr{B}$.

Corollary 1. If $Z \subseteq X$ then there exists a set $S$ containing $Z$ such that $S$ has property $(s)$, and if $E$ is any other set with property $(s)$ containing $Z$ then $S-E$ has property $\left(s^{0}\right)$.

Proof. Let $S$ be the set constructed in Theorem 3. If $T$ is any other Marczewski set containing $Z$, then $S-T$ will be totally imperfect and will therefore have property $\left(s^{0}\right)$. 
Now it has been shown that $B_{w}$ and $\mathscr{L}$ satisfy the conditions of both Theorem 1 and Theorem 2. Also it has been shown that $B_{r}$ and $U$ do not satisfy the conditions of either theorem, while $(s)$ meets the conditions of Theorem 1 but not the conditions of Theorem 2 .

\section{REFERENCES}

1. J. B. Brown and G. V. Cox, Classical theory of totally imperfect spaces, Real Anal. Exchange 7 (1982), 1-39.

2. C. Kuratowski, Topology Vol. I, New York-London-Warszawa: Academic Press, 1966.

3. J. C. Oxtoby, Measure and Category, New York-Heidelberg-Berlin: Springer-Verlag, 1980.

4. W. Sierpinski, Sur un problèm concernant les familles d'ensembles parfaits, Fund. Math. 31 (1938), 1-3.

5. E. Szpilrajn-Marczewski, Sur certains invariants de l'opération $(A)$, Fund. Math. 21 (1933), 229-235.

6. __ Sur une classe de fonctions de M. Sierpinski et la classe correspondante d'ensembles, Fund. Math. 24 (1935), 17-34.

7. J. T. Walsh, Marczewski sets, measure and the Baire property, Fund. Math., 129 (1988), 83-89.

1598 Tumbleweed Way, San Bernardino, California 92407 